\title{
E. Wilhelm and T. M. Letcher (eds): Heat capacities: liquids, solutions and vapours
}

\author{
RSC Publishing, Cambridge, 2010, 516 pp
}

\author{
John H. Dymond
}

Received: 7 June 2011/ Accepted: 20 July 2011/Published online: 26 August 2011

(C) Akadémiai Kiadó, Budapest, Hungary 2011

Chemical thermodynamics continues to be a subject of major importance for the understanding of phase transitions, chemical reactions, and (increasingly) biophysical processes, as well as its fundamental role in discussion of alternative future energy sources.

This volume, which is published under the auspices of the International Union of Pure and Applied Chemistry and the International Association of Chemical Thermodynamics, is the latest in a series of volumes to be published by these organisations that describe the state of the art instrumentation and experimental techniques for acquisition of thermodynamic property data for fluids, the critical evaluation of these data and the representation by equations of their dependence on variables such as temperature, pressure, and composition. The practical realization of these equations lies in their incorporation in computer simulation packages of chemical engineering processes.

The importance of heat capacities arises because they are intimately related to the temperature dependence of thermodynamic functions, and because they link thermodynamics with microscopic fluid structure. The topics covered in the 22 chapters in this volume include the underlying theory and the most important experimental techniques for direct and indirect determination of heat capacities; their application to gases at low and high pressure, to liquids at low and high pressure, to electrolyte and non-electrolyte solutions, to polymeric systems, to proteins and liquid crystals, plastic crystals and ionic liquids, and molten salts. There is a discussion of heat capacities in the critical region, of computer simulation studies of heat capacity effects associated with hydrophobic effects and of partial molar heat capacity changes of gases dissolved in liquids. The coverage by an international team of 35 authors, is wide-ranging though it should be mentioned that some topics, such as chemically reactive systems, are not included.

This book will be welcomed by researchers in chemical thermodynamics in academia and in the chemical industry.

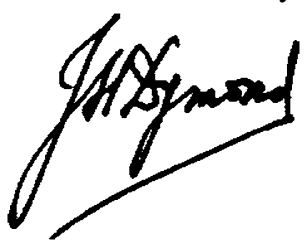

June 6, 2011

(J. H. Dymond)

J. H. Dymond ( $\square)$

Dunmore Cottage, Balfron G63 0TX, UK

e-mail: dunmorecot@tiscali.co.uk 